\title{
Fever with pain in bilateral shoulder joints
}

\author{
S Kapoor, A Mehtani, S Batra
}

Maulana Azad Medical

College and Associated

Lok Nayak Hospital,

Bahadur Shah Zafar

Marg, New Delhi,

India

S Kapoor

A Mehtani

S Batra

Correspondence to:

Dr Sumit Batra, B-39,

Adarsh Kunj Apartments,

Plot No-42, Sector-13,

Rohini, Delhi 110085, India

sumitbatra104@yahoo.com

Submitted 31 January 2001 Accepted 25 April 2001

A 15 year old male presented with a history of moderate fever with pain and limitation of movement of both shoulder joints for three weeks. On clinical examination the patient was febrile $\left(100^{\circ} \mathrm{F}\right)$ and there was tenderness around the shoulder and proximal half of his upper arm on both sides. There was periarticular muscle spasm but no signs of any collection. There was restriction of both active and passive movements. There were no other significant findings.

Investigations showed total leucocyte count $14.2 \times 10^{9} / 1$, with increased polymorphs (polymorphs $88 \%$, lymphocytes $10 \%$, monocytes $2 \%)$. Erythrocytic sedimentation rate was 63 $\mathrm{mm}$ /hour (Wintrobe's). Widal test was negative. All other blood investigations were within normal limits. Radiographs of both the shoulder joints were taken and are shown in fig 1 . Aspiration on the right side with an 18 gauge needle revealed a drop of pus, which was sent for culture and sensitivity examination. Aspiration on the left side was negative. The patient was operated on the right side. Adequate debridement was done and a bone biopsy specimen was taken; this showed signs of chronic inflammation.

\section{Questions}

(1) Describe the radiography findings.

(2) What is the diagnosis?

(3) What is the differential diagnosis?

(4) Discuss the treatment of this condition.

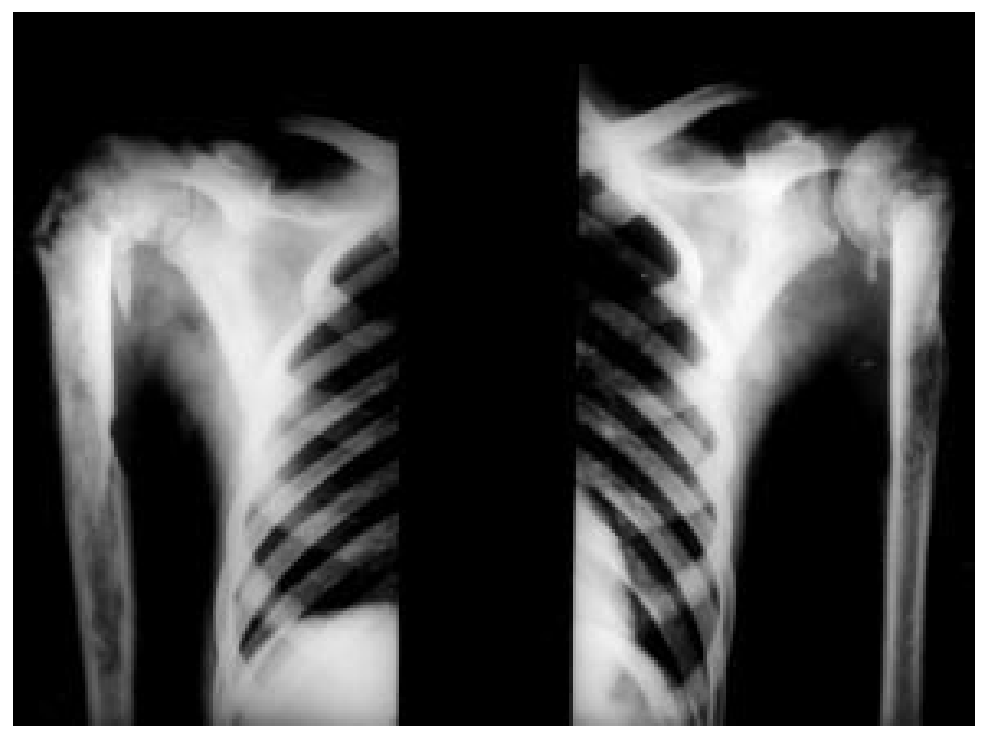

Figure 1 Radiographs of both the shoulder joints. 


\section{Answers}

QUESTION 1

The radiograph shows multiple osteolytic and destructive lesions simulating a permeative pathology, involving the upper half of the humerus on both sides. Evidence of pathological fracture of the surgical neck of humerus is seen on both sides with the left side showing more displacement.

QUESTION 2

The diagnosis is bilateral pathological fracture of the neck of the humerus secondary to pyogenic osteomyelitis.

QUESTION 3

The differential diagnosis is:

(1) Salmonella osteomyelitis usually involves multiple bones and can present as bilateral involvement of both humeruses. Patients with sickle cell disease are more prone to salmonella infection.

(2) Acute lymphocytic leukaemia in children can present with fever, bone pain, localised tenderness with lytic lesions in multiple bones, and can mimic acute osteomyelitis. Various fractures reported in cases of acute lymphocytic leukaemia include fracture of proximal tibia, distal radius, vertebral compression fractures, and bilateral slipped capital femoral epiphysis. Thomas et al reported one case of bilateral slipped upper humeral epiphysis in a patient with acute lymphocytic leukaemia with a radiographic picture resembling the case presented here. ${ }^{1}$ The diagnosis of leukaemia is established on the basis of investigations which show leucocytosis or leucopenia and the presence of immature leucocytes in the peripheral smear. The bone marrow picture confirms the diagnosis.

QUESTION 4

The treatment of such cases is difficult and prolonged. The most important step is to control the underlying infection. The treatment consists of drainage and debridement of the infection with prolonged immobilisation in association with antibiotic therapy.

On clinical suspicion, needle aspiration of the area of maximum tenderness is done to obtain subperiosteal fluid or is obtained directly from the metaphysis. If gross purulent material is obtained, surgical drainage should follow. ${ }^{2}$

The exact time for surgical debridement is controversial. One school of thought recommends delayed surgery to allow a strong involucrum to develop to provide support to the diseased bone.

Langenskiold showed good results in a 6 year old child by performing sequestrectomy of a necrotic femoral shaft after 10 months, which allowed the involucrum to develop. ${ }^{3}$

The other group recommends early surgery to allow faster healing by early eradication of infection. Daoud and Saighi-Bouaouina recommend much earlier debridement. ${ }^{4}$ They obtained healing in 33 of 34 patients with pathological fractures or pseudoarthrosis due to osteomyelitis. In patients with active infection, they performed sequestrectomy with debridement followed by antibiotic therapy for up to six months. Prolonged cast immobilisation was necessary. The mean healing time of fractures was five months in those patients with involucrum. Those patients with active infection without involucrum required debridement, antibiotics, and subsequent treatment with corticocancellous iliac grafts. The mean healing time was 8.7 months. Their patients without active infection and no involucrum were treated with prolonged immobilisation and cancellous bone grafts supplemented by fixation.

The recommended regimen for antibiotic therapy includes one week of parenteral antibiotics followed by 3-4 weeks of oral antibiotics if the organism is known, and serum bactericidal titres of the antibiotic are adequate to eradicate the organism. The peak bactericidal titre taken one hour after antibiotic dosage should be at least 1:8. Two per cent of patients never obtain good bactericidal levels because of poor gastrointestinal absorption. If oral drugs are inadequate or the organism is not isolated by culture, long term parenteral antibiotics must be used. The total length of therapy is based on the type of organism isolated and the patient's response to treatment. ${ }^{2}$

\section{Discussion}

Most patients with acute haematogenous osteomyelitis present with fever, pain, and localised tenderness at the site of infection. Most commonly involved bones in order of frequency are femur, tibia, humerus, fibula, radius, phalanges, calcaneus, ulna, ischium, metatarsals, and vertebral bodies. The erythrocyte sedimentation rate is commonly raised. The total leucocyte count is normal in $40 \%-75 \%$ of cases. ${ }^{2}$ Blood cultures are positive for the infecting organism in $40 \%-50 \%$ of cases ${ }^{5}$ and direct cultures of the site of infection are positive in $70 \%$ of cases. Most common organism is Staphylococcus aureus but in the very young child, Haemophilus influenzae B and Streptococcus pneumoniae have a significant prevalence. ${ }^{2}$ Rare causes include congenital rubella ${ }^{6}$ and congenital cytomegalic inclusion disease. ${ }^{7}$

In 1932, Capener and Pierce reviewed 1068 cases of osteomyelitis and found only 18 pathological fractures, 13 of which occurred in the femur. ${ }^{8}$ They thought these fractures were due to preventable causes, such as delayed recognition of the disease or inadequate treatment with eventual sequestration of the entire diaphysis. Other secondary factors considered were disuse atrophy of the bones, presence of a weak involucrum, and excessive surgical removal of either the diaphysial shaft or the surrounding involucrum.

Pathological fractures in osteomyelitis have been classified into two types by Glendhill and McIntyre. ${ }^{9}$ The first type is a supracondylar fracture of the femur that occurs secondary to osteoporosis and is related to immobilisation. The second type is a disruption of the diaphyseal-metaphyseal junction that is secondary to infection. These fractures generally 
tend to angulate and displace and commonly progress to delayed union and non-union.

Final diagnosis

Pathological fracture of the neck of the humerus secondary to pyogenic osteomyelitis.

1 Thomas LB, Forkner CE, Frei E III, et al. The skeletal lesions of acute leukemia. Cancer 1961;14:608-21.

2 Jackson MA, Nelson JD. Etiology and medical management of acute suppurative bone and joint infections in pediatric patients. F Pediatr Orthop 1982;2:313-23.

3 Langenskiold A. Femur remodelled during growth after osteomyelitis causing coxa vara and shaft necrosis. $\mathcal{f}$ Pediatr Orthop 1982;2:289-94.
4 Daoud A, Saighi-Bouaouina A. Treatment of sequestra, pseudoarthrosis and defects in the long bones of children who have chronic hematogenous osteomyelitis. f Bone foint Surg Am 1989;71:1448-67.

5 Septimus EJ, Musher DM. Osteomyelitis: recent clinical and laboratory aspects. Orthop Clin North Am 1979;10:34759.

6 Sacks R, Habermann ET. Pathological fracture in congenital rubella: a case report. F Bone foint Surg Am 1977;59:557tal

7 Smith RK, Specht EE. Osseous lesions and pathological fractures in congenital cytomegalic inclusion disease: a report of a case. Clin Orthop 1979;144:280-3.

8 Capener M, Pierce KC. Pathological fractures in osteomyelitis. F Bone foint Surg Am 1932;14:501-10.

9 Glendhill RB, McIntyre JM. Various phases of pediatric osteomyelitis. AAOS Instructional Course Lectures 1973;22: 